\title{
LA EPISTEMOLOGÍA DE LA INVESTIGACIÓN CIENTIIFICA EN LAS CIENCIAS SOCIALES Y LAS HUMANIDADES
}

\section{THE EPISTEMOLOGY OF SCIENTIFIC INVESTIGATION IN SOCIAL SCIENCES AND HUMANITIES}

\author{
Paolo Musso* \\ Recepción: 08/Noviembre/2016 \\ Aceptación: 07/Marzo/2017.
}

\begin{abstract}
RESUMEN
En nuestro tiempo muy a menudo parece que las ciencias sociales, y más aún las humanidades, se encuentran atrapadas entre dos alternativas opuestas, pero igualmente insatisfactorias: o conformarse completamente al método de la ciencia natural (lo que las empuja hacia un deletéreo reduccionismo, que olvida exactamente las propiedades más características de sus objetos) o renunciar a la ambición de ser formas de auténtico conocimiento (lo que las empuja hacia un igualmente deletéreo irracionalismo, que de hecho las vuelve inefectivas y luego inútiles). Sin embargo, exactamente el correcto entendimiento del método experimental, de su naturaleza y de sus límites nos permite fundamentar la plena legitimidad de otras formas de auténtico conocimiento además de la ciencia natural, de la cual difieren, no por su naturaleza, sino solo por el grado de certeza que pueden alcanzar, ya que usan métodos distintos que dependen de sus distintos objetos y no son completamente iguales ni completamente diferentes al método experimental, sino análogos.
\end{abstract}

\section{PALABRAS CLAVE}

Galileo Galilei, método científico, ciencias sociales, humanidades, sistemas complejos, sentimiento, objetividad, intersubjetividad.

\begin{abstract}
Nowadays, it seems very often that social sciences, and even more the humanities, are trapped between two opposite but equally unsatisfactory alternatives: either to conform completely to the method of natural science (which push them towards a deleterious reductionism that disregards the most characteristic properties of their objects) or to give up the ambition of being forms of true knowledge (which push them towards an equally deleterious irrationalism that turns them ineffective and therefore useless). However, a correct understanding of the experimental method, of its nature and its limits, enables us to demonstrate the full legitimacy of other forms of true knowledge besides natural science, from which these other forms differ, not in regards to their nature, but in regards to the degree of certainty they can reach, since they use different methods that are dependent on their different objects and that are neither completely identical nor completely different from the experimental method, but analogous to it.
\end{abstract}

\section{KEY WORDS}

Galileo Galilei, scientific method, social sciences, humanities, complex systems, feeling, objectivity, intersubjectivity.

* Docente de la Università degli Studi dell'Insubria di Varese (Italia): paolo.musso@uninsubria.it 


\section{La relación con la ciencia natural}

Cuando se habla del método de la investigación en cualquier campo del conocimiento humano no se puede evitar hacer una comparación con el método de la ciencia natural, no sólo porque, por su gran efectividad, se ha vuelto un punto de referencia imprescindible, sino también porque a menudo surge el problema de establecer si (y en el caso positivo hasta qué punto) dicho método puede ser aplicado en otros ámbitos, como por ejemplo en las ciencias humanas y sociales.

El método de la ciencia natural, como todos saben, fue definido por Galileo Galilei, de una manera extremadamente clara, simple y sobretodo definitiva, en el siglo XVII y sigue siendo idéntico hoy en día, después de 4 siglos, lo que es casi increíble, más aún si consideramos que Galileo nunca escribió un ensayo sistemático sobre del tema, sino que sus principios metodológicos se encuentran esparcidos en varios pasajes de sus libros y a veces incluso de sus cartas.

Vamos por tanto a ver cuáles son los 4 principios básicos del método científico según Galileo,' acerca de los cuales todos los filósofos y los historiadores de la ciencia más o menos están de acuerdo (lo que acontece muy raramente y demuestra una vez más el excepcional nivel de claridad que supo alcanzar Galileo al propósito):
1) No buscar la esencia de las cosas, sino limitarse a estudiar algunas propiedades.

2) No solo genérica observación, sino experimento.

3) Uso de la matemática.

4) Ningún principio de autoridad.

Y ahora vamos a analizar cada punto un poco más en detalle.

1) En primer lugar cabe subrayar que, aunque obviamente todos los 4 principios tengan que ser aplicados juntos para que el método pueda funcionar, el primero es el más importante. En efecto, desde la Grecia antigua hasta Galileo todos siempre intentaron hacer la ciencia según el método deductivo (o, como se dice en el argot de los filósofos, a priori), ${ }^{2}$ intentando definir por medio de la pura razón las esencias de las cosas y luego deducir de ellas todos los particulares: pero siempre fracasaron. Hay que decir que, en un sentido, los griegos fueron las víctimas de su propio éxito. En efecto, dicho método, lejos de ser bárbaro, obscurantista o supersticioso, demostró tener una extraordinaria eficacia en muchas disciplinas, como la metafísica, la lógica, la matemática y, sobre todo, la geometría, gracias a los Elementos de Euclides: por tanto, es del todo comprensible que los griegos y sus seguidores medioevales hayan pensado de usarlo también en el campo de la ciencia natural. ${ }^{3}$ Sin embargo, no todo

1 Para todo lo que se dice en este párrafo y en el siguiente, véase Musso (2011), § 1.13.

2 Pues ya se ha vuelto de uso común, yo también usaré esta expresión, que es tomada de la obra de Kant, pese a que no sea nada precisa, porque implica la idea de un sistema conceptual construido de manera completamente independiente de la experiencia sensible, según el estilo (equivocado) típico de la filosofía moderna. En cambio, la ciencia aristotélica era independiente de la experiencia sensible sólo en el sentido de que afirmaba que era posible llegar a conocer las esencias de las cosas a través de la pura reflexión, sin necesidad de hacer ni cálculos ni experimentos: sin embargo, dicha reflexión era acerca de la experiencia sensible y por esto Aristóteles valoraba mucho la observación (pero que no es la misma cosa que el experimento, como veremos de inmediato).

3 Los Elementos de Euclides eran una obra tan moderna que su estructura axiomática será mejorada por primera vez solo después de 22 siglos, por David Hilbert en su Grundlagen der Geometrie (Hilbert 1899). 
lo que a primera vista parece justo lo es realmente.

El golpe de genio de Galileo fue exactamente el haber entendido primero que en el caso de la ciencia natural (y sólo en este caso) era necesario invertir el método, empezando por los aspectos más simples y particulares, que conocemos por medio de la experiencia sensible, para después llegar a los más profundos y generales, que en cambio pueden estar (y muchas veces están realmente) más allá de la experiencia. Y esto es el famoso texto de Las manchas solares donde se encuentra la primera formulación de la inversión metodológica galileana: «Por lo tanto, o queremos intentar de penetrar especulando la esencia verdadera e intrínseca de las sustancias naturales; o queremos conformarnos con descubrir algunas de sus propiedades. Buscar la esencia, yo creo que es un intento inútil tanto en el caso de las sustancias más simples como de las más lejanos y celestes: y me parece que soy igualmente ignorante sobre la sustancia de la Tierra que, de la Luna, de las nubes y de las manchas del Sol; ni veo que comprendiendo estas sustancias cercanas tenemos otra ventaja que el número de las propiedades, pero todas igualmente desconocidas. [...] Pero si queremos limitarnos al aprender algunas propiedades, no me parece que sea imposible descubrirlas también en los cuerpos más lejanos de nosotros, no menos que en los cercanos» (Galileo 1613: 187-188).

Es importante entender que dicha limitación solo es metodológica. Galileo no era ni un fenomenista (es decir, uno que piensa que solo podemos conocer lo que aparece y no lo que realmente es) ${ }^{4}$ ni, mucho menos, un escéptico. Véase, por ejemplo, este texto, también tomado de Las manchas solares: «Los nombres y las propiedades tienen que adaptarse a la esencia de las cosas, y no la esencia a los nombres; porque antes fueron las cosas, y después los nombres» (Galileo 1613a: 97). O este de los Diálogos sobre los dos máximos sistemas, hasta más contundente: «SALVIATI - Usted yerra, señor Simplicio; usted debe decir que cualquiera sabe que se llama gravedad. Pero yo no le pregunto sobre el nombre, sino sobre la esencia de las cosas» (Galileo 1632: 260). ¿Hay contradicción? No, porque Galileo no rechaza por nada la búsqueda de la esencia, que consiste simplemente en las propiedades fundamentales de una cosa, o sea, las que hacen que sea lo que es y de las cuales por tanto derivan todas las otras propiedades suyas. Ahora bien, no cabe duda que esto es lo que la ciencia siempre ha intentado (y en parte también logrado) descubrir. Por otro lado, esto lo demuestra también el testimonio de toda su vida, pues si Galileo sufrió el proceso, fue exactamente porque nunca aceptó presentar sus ideas solo como hipótesis (lo que estaba permitido), sino como conocimientos verdaderos de las cosas, es decir, justamente, de su esencia. Sin embargo, con Galileo el conocimiento de la esencia se convierte en el punto de llegada de la investigación científica (que solo se puede lograr por grados, un poquito a la vez) en lugar de ser su punto de partida, como era para los antiguos.

4 En efecto esta idea, hoy muy común, no solo es falsa, sino también antihistórica, pues dicha distinción entre propiedades y esencia solo nació con Kant y su famosa separación entre, exactamente, los "fenómenos" y las "cosas en sí". Según Aristóteles, en cambio, las propiedades sensibles (por lo menos las más importantes, los "accidentes propios e inseparables") son parte de la "esencia" de una cosa, pese a que la esencia no se reduce a un conjunto de propiedades sensibles (exactamente como, por otro lado, tampoco las teorías científicas, que para Galileo tienen que descubrir qué son realmente las cosas, se reducen a un conjunto de enunciados empíricos, como querían los neopositivistas). 
Además, cabe subrayar que, aunque el método de Galileo no sea deductivo, tampoco es inductivo, como muy a menudo erróneamente se dice: en efecto, el inductivismo no es una alternativa real al deductivismo, más bien, es la otra cara de la misma moneda, ya que sigue oponiendo razón y experiencia, mientras que el método de Galileo se basa en la unidad indisoluble de las dos.

Muchos historiadores y epistemólogos no están de acuerdo conmigo y eligen a uno u otro de los demás principios como el más importante, pero en efecto hay una estrecha relación lógica entre los 4 principios galileanos, que están concatenados uno a otro de modo tal que los demás dependen todos, directamente o indirectamente, del primero, como ahora veremos.

2) El segundo punto del método galileano es, como hemos dicho, el experimento en cambio de la simple observación. ${ }^{5}$ Ahora bien, el experimento se diferencia de la simple observación porque es artificial y orientado, lo que pide una intervención activa del científico. Pero esto solo es posible exactamente porque tiene como su tarea evidenciar y estudiar "algunas propiedades", y por tanto se basa en el primer principio. Además, esto es también lo que hace que los experimentos sean repetibles, lo que es necesario para el control intersubjetivo, en que se basa la objetividad de todo conocimiento. En efecto, en nuestro mundo ninguna situación nunca se repite exactamente idéntica: luego, si fuese necesario considerar todos los factores, se debería concluir que ningún experimento es nunca realmente repetible, mientras si hacemos referencia solo a "algunas propiedades" la repetitividad es posible. ${ }^{6}$

3) El tercer punto del método galileano es el uso de la matemática para escribir las leyes naturales que el experimento tendrá que verificar. Sin embargo, también la matemática, para poderse aplicar al estudio de la naturaleza, necesita un objeto definido de manera precisa y no ambigua: por ejemplo, las leyes del movimiento solo describen la trayectoria de un cuerpo, no su color o su temperatura. Luego, también este principio para funcionar tiene que limitarse al estudio de "algunas propiedades" y por lo tanto se basa en el primero.

4) Por fin, Galileo rechaza el principio de autoridad, pero no porque fuese un anarquista o un rebelde, como hoy es de moda pensar, sino solo porque para él en la ciencia hay una autoridad superior a la humana, que todos pueden interrogar a través de la matemática y el experimento, que es como una pregunta hecha a la naturaleza y, a través de esa, últimamente a Dios mismo, que es su Creador. Y como la matemática y el experimento a su vez se basan en el primer principio, entonces también este cuarto principio depende del primero, que por tanto es realmente $e l$ principio fundamental del método galileano. Sin embargo, hay que precisar que todo esto solo vale en principio: en cambio, en la práctica está claro que, si cada científico

5 Aunque hay que decir que Galileo casi nunca usa el término "experimento" (solo 3 veces en toda su obra: dos en El ensayador y una en los Discursos y demostraciones matemáticas sobre dos nuevas ciencias), sino, según la costumbre de su tiempo, el término "experiencia". Sin embargo, en base al contexto se entiende claramente que está hablando del experimento en el sentido moderno del término.

6 En efecto, muchos le objetaron a Galileo que estudiando propiedades aisladas se alteraba la realidad y hoy sabemos que en parte tenían razón, pues esto es exactamente lo que pasa en los sistemas no lineales (véase más delante): afortunadamente, el mundo no está hecho todo así, pues en este caso la ciencia sería imposible, pero Galileo esto no lo sabía, solo podía esperarlo. iSe necesitaba realmente una gran fe en la inteligibilidad de lo real (que a Galileo le venía de su fe cristiana) para proponer un método de este tipo! 
tuviese que empezar todo el trabajo desde el principio, el progreso científico sería imposible. Por tanto, tampoco la ciencia puede prescindir del método del conocimiento por fe (que continuamente usamos en nuestra vida de cada día y que consiste en creer algo no porque lo hemos visto personalmente, sino en base a lo que dice un testigo fiable) y luego tampoco del principio de autoridad, en que se basa la posibilidad de la enseñanza y que por tanto se vuelve cada vez más importante con el incremento del número, de la extensión y de la profundidad de nuestros conocimientos. Luego, hay como una tensión entre el aspecto teórico y el práctico, que es inevitable y que cada científico tiene que manejar lo mejor que puede, intentando entender cuándo es oportuno fiarse con los ojos cerrados y cuándo, en cambio, es mejor controlar personalmente. Es interesante notar que en todo esto juega un papel importante no solo la experiencia profesional, sino también la humana, porque en la decisión de fiarse no entra en juego solo un juicio técnico sobre la preparación científica del otro, sino también un juicio sobre su fiabilidad como persona (método de la certeza moral): luego, al fin y al cabo, en la realidad concreta, tampoco el método científico, por más objetivo e impersonal que sea, puede prescindir completamente del factor humano.

\section{El pluralismo metodológico galileano}

Como Galileo dice claramente en el texto de Las manchas solares que hemos recién citado, así como en muchos otros, su método vale solo en el caso "de las sustancias naturales", o sea de los "cuerpos". Es decir, para Galileo, diferentemente a los antiguos, ${ }^{7}$ no hay un método único del conocimiento que va bien para todo: el método depende del objeto, luego objetos diferentes piden métodos diferentes (pluralismo metodológico, no reduccionismo).

En particular, la ciencia nace de una precisa y consciente autolimitación metodológica a los aspectos medibles de la realidad: luego, el mismo método científico, si bien entendido, es el mejor antídoto contra el cientificismo, sin ninguna necesidad de negar el valor cognoscitivo de la ciencia, como en cambio lo hace la epistemología moderna (y como, lamentablemente, lo piensan también muchos filósofos católicos). ${ }^{8}$ En efecto, el reconocimiento de la legitimidad, más bien, incluso de la necesitad de la existencia de otras formas de conocimiento es un factor constitutivo de la ciencia misma y no algo que se le ha intentado imponerle indebidamente desde el exterior en un momento sucesivo. Y, de hecho, Galileo, pese a que nunca desarrolló una reflexión sistemática sobre las formas de conocimiento diferentes a la científica, que era la única que le interesaba y de la cual quería ocuparse, de todos modos, reconoció explícitamente el valor de al menos dos otras formas de conocimiento además de la ciencia experimental y de la matemática: la teología, basada en la Revelación divina, y el arte, basada en la capacidad de ensimismarían en los sentimientos de los demás.

7 Es verdad que también para Aristóteles existían diferencias entre los métodos con los cuales se podían conocer los principios de las varias ciencias y los niveles de certeza a los cuales podían llegar. Sin embargo, el esquema de fondo siempre era el mismo: en primer lugar, se debía establecer los principios básicos, para después deducir de esos todos los detalles.

8 Muy distinto, en cambio, es el pensamiento de Benedicto XVI, que, en una charla dictada cuando todavía era solo Cardenal, tras hablar de dicha epistemología relativista agregó: «Sería absurdo construir en base a estas afirmaciones una apresurada apologética. La fe no crece a partir del resentimiento y del rechazo de la racionalidad, sino de su fundamental afirmación y de su inscripción en una racionalidad más ancha» (Ratzinger 1992: 79). 


\section{El método de las ciencias humanas y sociales}

Ahora bien, como el hombre no es un puro espíritu, sino tiene un cuerpo y actúa dentro de un mundo material, la autolimitación del método galileano a los aspectos medibles de la realidad no impide su aplicación a las ciencias humanas y sociales (historia, política, economía, sociología, medicina, psicología, etc.). Sin embargo, en este caso sufre 2 diferentes limitaciones.

1) En primer lugar, pues en el hombre hay también algo que no se reduce a su cuerpo y luego no es medible, la aplicabilidad del método galileano en este campo solo y siempre será parcial.

2) Además, gracias a los descubrimientos del fenómeno del caos determinista y de la geometría fractal por obra respectivamente de Edward Lorenz (1963) y Benoît Mandelbrot (1975), hoy sabemos que los sistemas complejos, ${ }^{9}$ entre los cuales estamos incluidos también nosotros los seres humanos y los varios sistemas sociales en que vivimos, nunca son completamente predecibles, ni siquiera en la parte que puede ser estudiada por medio del método galileano, cuya aplicabilidad en este campo, por tanto, siempre será también incompleta.

Luego, por más cuidadosamente que uno pueda investigar, en este tipo de ciencias siempre y necesariamente quedará algo indeterminado, que solo se podrá tratar de entender a través de un análisis cualitativo, que en parte dependerá de las ideas, las intuiciones y - digámoslo sin miedo - de la genialidad personal de cada investigador, pero en parte también de valores y conceptos tomados de las humanidades (filosofía, ética, antropología, arte, religión, etc.), que, al fin y al cabo, no son nada más que el conjunto de las ideas e intuiciones que han tenido al propósito los investigadores del pasado y que forman como un "reservorio" en que pueden útilmente basarse los investigadores del presente.

Sin embargo, esto nos lleva a plantear el problema del valor cognoscitivo de las propias humanidades, o sea, de toda aquella parte de nuestra cultura a la cual el método galileano no se puede aplicar por nada, ni siquiera parcialmente.

\section{Las dos culturas}

En base a todo lo anterior, debería quedar claro que, como la ciencia no puede agotar toda la realidad, es por lo menos posible la existencia de tipos de conocimiento distintos al científico, o sea, de conocimientos no científicos. El problema es si se trata de auténticos conocimientos o de meras expresiones de sentimientos ${ }^{10}$ subjetivos, como opina casi toda la cultura hodierna, sobre todo por la influencia del positivismo y de la filosofía analítica, que en gran parte es su directa emanación, aunque de verdad ambos derivan de una tradición mucho más antigua, que se puede remontar a Descartes y que ya desde hace tiempo se han vuelto mentalidad común.

9 Acá el término "complejo" es entendido no de una manera genérica, o sea, como más o menos equivalente a "complicado", sino en un sentido estrictamente técnico, o sea, como equivalente a "sistema no lineal" o "sistema fractal". Todos los seres vivos (no solo los seres humanos) y todos los sistemas sociales son no lineales o fractales, así como también muchos (pero no todos) los sistemas físicos inorgánicos.

10 Acá el término "sentimiento" no quiere indicar solo los sentimientos para las otras personas, sino cualquier tipo de emoción generado en nosotros por el impacto con la realidad. 
En efecto, hoy en día no son sólo los positivistas y los cientificistas que lo piensan así, sino que muy a menudo también los que abiertamente se oponen a sus ideas finalizan con aceptarlas implícitamente, apreciando la ciencia solo por sus logros prácticos y opinando que el sentido de la vida se deba buscar por otros caminos, como el arte, la filosofía, la poesía y la religión, pero entendidas de un modo sustancialmente no cognoscitivo y luego últimamente irracional. Incluso los científicos que rechazan el reduccionismo y que en su trabajo de investigación aprecian también el aspecto de desinteresada contemplación de la verdad y de la belleza que se encuentran en la naturaleza, tienen generalmente cierta tendencia a pensar que la ciencia no tiene mucho que ver con el sentido de la vida y, más generalmente, con una visión global de la realidad, que ellos también buscan más o menos por los mismos caminos y con la misma postura de la gente común. ${ }^{11}$

Ahora bien, si es verdad que la confusión entre los diferentes niveles de la realidad es un error grave y peligroso, también su total separación es igualmente deletérea, pues al fin y al cabo siempre se trata de diferentes niveles de la misma realidad, así como siempre es la misma persona la que conoce dicha realidad, aunque a través de una pluralidad de métodos. Luego, si la justa distinción entre esos se convierte en completa separación o, peor aún, en abierta oposición, será inevitable caer en una postura esquizofrénica, en que hay algo como una "doble verdad", pues la razón dice una cosa y el corazón dice el opuesto (lo que puede incluso causar, y a veces en algunas personas causa realmente, la esquizofrenia en el sentido literal del término).

Sin embargo lamentablemente, parece que exactamente ésta sea la postura que desde hace tiempo se ha afirmado, hasta el punto que ya en 1959 el físico y escritor inglés Charles Percy Snow en su célebre libro Las dos culturas había llegado a decir que precisamente la falta de comunicación entre la cultura científica y la humanista era la causa principal que impide solucionar los problemas básicos de la humanidad. En efecto, dicha fractura tiene consecuencias particularmente graves en el caso de las ciencias humanas y sociales, que, como ya hemos dicho, deberían basarse en una mezcla de método experimental y de valores y conceptos tomados de las humanidades: por consiguiente, el rechazo a reconocer a las humanidades el estatus de auténtico conocimiento, dejando el método experimental como unico instrumento aceptable para las ciencias humanas y sociales, las ha inevitablemente empujado hacia el cientificismo, con todas las catastróficas consecuencias que estamos viendo, especialmente en economía, donde la deriva cientificista es particularmente evidente, ${ }^{12}$ pero en parte también en

11 Esto no debe sorprendernos, pues, al fin y al cabo, pese a todas sus extrañas especializaciones, los científicos son gente común, es decir, hombres de su tiempo, que sufren como todos los otros la influencia de las mismas modas y tendencias culturales: como lo dijo muy bien recientemente el actual Director de la Investigación del CERN de Ginebra, Sergio Bertolucci, «en el CERN tenemos la misma variedad de personas que se puede encontrar en un supermercado» (Bertolucci 2014: 57).

12 Bastaría con considerar que todas las actuales crisis financieras son muy amplificadas por el uso cada vez más frecuente de programas automáticos, que actúan independientemente de cualquier control humano. Pero hay mucho más, pues el origen profundo de dichas crisis está en una concepción mecánica del ser humano y de su manera de actuar, que pretende ser "científica", pero de verdad solo es (groseramente) cientificista (cf. Musso 2011: cap. 2 y 3). No por nada, Benedicto XVI en un célebre discurso al Pontificio Consejo para los Laicos del 25 de noviembre de 2011 había afirmado que la 
medicina y en psicología, donde las tendencias reduccionistas y la escasa consideración del factor humano están creciendo de un modo preocupante, arriesgando incluso disminuir la eficacia de los grandes avances que se están dando al nivel técnico.

Es por tanto esencial entender que no se trata de un destino fatal e inevitable, sino que todo el problema nace de un equívoco.

\section{Del dualismo metafísico al pluralismo orgánico}

Dicho equivoco consiste en el malentendido del sentido profundo de la objetividad científica, ${ }^{13}$ que es identificada con una supuesta "impersonalidad" de la ciencia misma, mientras que de verdad coincide simplemente con el requisito de la repetitividad de los experimentos. En efecto, todo lo que se requiere para que cierto resultado pueda ser considerado objetivo es que el experimento que lo ha producido pueda ser repetido por quienquiera, prescindiendo por tanto de las características particulares de este o aquel sujeto humano, pero por nada del sujeto humano como tal, sin el cual ningún experimento tendría sentido. Más bien, en realidad el requisito de la repetitividad de los experimentos se basa exactamente en el supuesto de la existencia de una naturaleza humana común a todos los sujetos particulares, que siempre es básicamente la misma en todos los seres humanos, en todo tiempo y lugar, a pesar de todas las diferencias culturales, religiosas, políticas, educativas, etcétera. Si así no fueran las cosas, no sería evidentemente posible para sujetos completamente diferentes llegar a los mismos resultados: y en efecto el motivo por el cual la epistemología moderna ha llegado a la tesis de la inconmensurabilidad de las teorías y luego al relativismo es exactamente porque no admite que la razón humana exista antes e independientemente de cualquier esquema conceptual.

Luego, cualquier forma de la cultura humana puede ser considerada un conocimiento objetivo, bajo la única condición de estar en grado de indicar algunos criterios de evaluación que sean aplicables por cualquier ser humano, en todo tiempo y lugar, independientemente de sus características individuales. Imponer ulteriores condiciones significaría simplemente pretender de aplicar el método científico más allá de su propio ámbito, que, como hemos visto, es limitado por su misma naturaleza.

En otras palabras, el criterio decisivo para poder afirmar que una creencia cualquiera es un auténtico "conocimiento" es si hay la posibilidad de una verificación intersubjetiva. En cambio, no se requiere que dicha verificación siempre sea alcanzada a través del método experimental, aunque, claro, esto implica que tampoco tendrá su mismo grado de fiabilidad.

Esto significa que entre el método científico y los varios métodos de las otras formas de conocimiento no hay ni identificación (como sostiene el racionalismo) ni incomunicabilidad (como sostiene el relativismo), sino una relación de analogía.

Sin embargo, hay que tomar conciencia de que todo esto implica la superación del

actual «es crisis de significado y de valores, antes de ser crisis económica y social». El problema es que entonces todos coincidieron, llegando en algunos casos incluso a decir que «hoy en día el único auténtico economista que hay en el mundo es el Papa), pero después, apenas lo peor pasó (o pareció haber pasado), todos lo olvidaron y ya están de nuevo actuando exactamente al igual que antes.

13 Para todo lo que se dice en este párrafo y en el siguiente, véase Musso (2011), cap. 9. 
dualismo metafísico de derivación cartesiana en que se basa toda la filosofía moderna y su reemplazo por un pluralismo orgánico, en el cual ya no hay una insuperable diferencia de naturaleza entre lo que es objetivo y pertenece a la razón y lo que es subjetivo y pertenece al sentimiento, sino, en cambio, solo una diferencia de grado entre diferentes tipos de objetividad característicos de diferentes tipos de conocimientos, en cada uno de los cuales tanto la razón como el sentimiento están involucrados y cooperan activamente, aunque de formas y maneras diferentes, en dependencia de sus diferentes objetos.

\section{Moralidad y dinámica del conocimiento}

Luego, la cuestión no es eliminar el sentimiento del proceso del conocimiento (lo que sería no sólo imposible, sino hasta dañino), sino velar para que siempre esté en su lugar, actuando como una lente que nos acerca la realidad y no como una pared que nos la esconde: es decir, se necesita hacer sitio para la moralidad (entendida en este preciso sentido) al interior de la misma dinámica del conocimiento.

En efecto, es verdad que el método científico, gracias a su rigor y precisión, nos educa a una lealtad con la experiencia que en otras cuestiones menos exactamente definibles es mucho más difícil lograr (y por esto la ciencia puede tener también una importante función educativa). Sin embargo, incluso desde este punto de vista en realidad sólo se trata de una diferencia de grado y no de naturaleza, exactamente como hemos dicho en lo anterior, pues, como demuestra por ejemplo la trágica vicisitud de Boltzmann (así como muchas otras que se podrían citar), en efecto la moralidad, o sea, la tensión hacia la verdad ("amar a la verdad más que a uno mismo") siempre es necesaria en la dinámica del conocimiento, en todo nivel, también en la ciencia. Por otra parte, ya hemos visto que incluso el método científico en su normalidad (y no solo en estos casos "patológicos"), cuando se aplica al mundo real, no puede prescindir completamente del método de la certeza moral y luego del compromiso de toda la persona.

En la ciencia natural esto es más fácil, pero solo porque por un lado estamos frente a evidencias más fuertes, y por otro lado es más raro que sean involucrados sentimientos e intereses personales. Como ha escrito el gran físico y teólogo inglés John Polkinghorne: «La ciencia no es radicalmente diferente a las otras formas de investigación racional humana. Esa también requiere un riesgo desde el punto de vista intelectual, el compromiso con una teoría potencialmente modificable. Esa también implica juicios fiables, pero no completamente ciertos. El poder superior de la ciencia en solucionar problemas no consiste en su invencible certitud, sino en la apertura a la verificación que le deriva del estar implicada con aspectos de la realidad lo suficientemente impersonales como para ser accesibles a investigaciones repetidas y controles experimentales») (Polkinghorne 1996: 87).

Sin embargo, cuando, pese a todo esto, ocurre que, por alguna razón, los sentimientos personales entran en juego, también para los científicos surge el problema de manejarlos correctamente y si no lo logran la objetividad de la ciencia se encuentra en peligro, aunque en el largo plazo siempre acaba con imponerse, gracias a la intrínseca capacidad de autocorrección que tiene gracias a su método.

Los conocimientos de tipo no científico no tienen esta ventaja, pero esto no significa que en esos la verdad 
sea inalcanzable: solo significa que para alcanzarla se necesita un mayor compromiso de nuestra libertad.

\section{REFERENCIAS}

Bertolucci Sergio (2014). «La ricerca, motore vitale». Missioni Consolata, n. 12, pp. 50-58.

Galilei Galileo [1613] Istoria e dimostrazioni intorno alle macchie solari e loro accidenti, Roma. Giacomo Mascardi. In Galileo Galilei (1890-1909), Edizione Nazionale delle Opere di Galileo Galilei, Firenze, Giunti Barbera, vol. V, pp. 72-249.

Galilei Galileo (1632). Dialogo sopra $i$ due massimi sistemi del mondo, tolemaico e copernicano. Giovanni Battista Landini, Firenze. In Galileo Galilei (1890-1909), Edizione Nazionale delle Opere di Galileo Galilei, Firenze, Giunti Barbera, vol. VII, pp. 23-546.

Hilbert David (1899). Grundlagen der Geometrie. Stuttgart: Teubner.
Lorenz Edward (1963). «Deterministic non periodic flow». Journal of the Atmospheric Sciences, vol. 20, pp. 130-141.

Mandelbrot Benoît (1975). Les objets fractals. Paris: Flammarion.

Musso Paolo (2011). La scienza e l'idea di ragione. Scienza, filosofia e religione da Galileo ai buchi neri e oltre. Milano: Mimesis.

Musso Paolo (2012). Formas de la epistemología contemporánea. Entre realismo y anti-realismo. Lima: Fondo Editorial UCSS.

Polkinghorne John (1996). Beyond science: the wider human context. Cambridge: Cambridge Univesity Press.

Ratzinger Joseph (1992). Svolta per l'Europa? Chiesa e modernità nell'Europa dei rivolgimenti, Roma: Edizioni Paoline.

Snow Charles Percy (1959). The two cultures. Cambridge: Cambridge University Press. 